\title{
Investigating Signal Loss due to a Carotid Artery Stent in 3D-TOF-MRA
}

\author{
Hiroshi Kato*, Norio Ootani, Kentaro Abiru, and Mika Okahara
}

\begin{abstract}
Purpose: In this study, we investigated the factors of signal loss out because of the presence of a stent and optimized imaging parameters for improvement in depiction ability.

Methods: We investigated the relationship between the stent type and magnetic susceptibility effect by measuring the signal value between the inside and outside of the stent with different $\mathrm{Bw}$ and TE for two different kinds of stents respectively. Similarly, flip angles were changed for two different kinds of stents respectively to the signal intensity between the inside and the outside of the stent was measured, in which examine the relationship between the stent type and the Ernst angles in RF-shielding effect. The conventional imaging parameters and the optimum imaging parameters for each stent obtained from the result of the phantom experiment were examined. Optimized 3D time-of-flight MR angiography (3D-TOF-MRA) was performed and compared with conventional 3D-TOF-MRA and computed tomography angiography (CTA).
\end{abstract}

Results: The influence of the magnetic susceptibility effect is small in the central part of Carotid Wallstent and in PRECISE, and large in the Carotid Wallstent at the both ends. The influence of RF-shielding effect was large at PRECISE, where the Ernst angle was greatly shifted while the effect is no longer influenced at Carotid Wallstent. Both Carotid Wallstent and PRECISE made imaging capability improved by optimizing the imaging parameters.

Conclusion: During clinical imaging of patients post-carotid artery stenting (CAS) using our protocol, the ability to visualize blood vessels was improved. Thus, we demonstrated that the ability of 3D-TOF-MRA post-CAS was improved via optimizing imaging parameters.

Keywords: carotid artery stenting, RF-shielding effect, 3D time-of-flight magnetic resonance angiography, magnetic susceptibility effect

\section{Introduction}

\section{Background}

Carotid artery stenting (CAS) using a protection device to prevent distal embolism has increased for cases with vulnerable plaque. 3D time-of-flight MR angiography (3D-TOFMRA) is one of the tool to evaluate the patency of vessels, which are stented. However, many reports showed that the utilization of the stent resulted in a signal loss at the site of the

Radiology Department, Federation of National Public Service Personnel Mutual Aid Associations Shinbeppu Hospital, Beppu, Oita, Japan

${ }^{*}$ Corresponding author: Radiology Department, Federation of National Public Service Personnel Mutual Aid Associations Shinbeppu Hospital, 3898, Tsurumi, Beppu, Oita 874-0833, Japan. Phone: +81-977-22-0391, Fax: +81-977-26-4170, E-mail: hiroshi380905@yahoo.co.jp

(C)2020 Japanese Society for Magnetic Resonance in Medicine

This work is licensed under a Creative Commons Attribution-NonCommercialNoDerivatives International License.

Received: June 26, 2019 | Accepted: July 16, 2020 stent upon evaluation with 3D-TOF-MRA. ${ }^{1-4}$ Although it was understood that signal loss was caused by magnetic susceptibility and radio frequency RF-shielding effect, ${ }^{5-9}$ so far there have been a few reports on examining details of factors of in signal loss within the stent.

\section{Purpose}

The purpose of this study is to investigate the factors of signal loss owing to the presence of a stent and to optimize imaging parameters for improvement in depiction ability.

\section{Materials and Methods}

A 1.5T MRI imaging system (Optima MR450w, GE Healthcare, Barrington, IL, USA) was used in our study. The coil was quadrature detection (QD) head coil for phantom experiment, and Head Neck Unit for clinical examination.

This study is a fundamental experiment using a phantom and an image evaluation by a clinical case. 


\section{Phantom study (optimization of imaging parameters setting) \\ Phantom preparation}

The artifacts of two different stents were evaluated. These stents (PRECISE [diameter: $10 \mathrm{~mm}$ ] [Johnson \& Johnson, New Brunswick, NJ, USA] and Carotid Wallstent [diameter: $10 \mathrm{~mm}$ ] [Boston Scientific, Natick, MA, USA]) were placed in plastic boxes filled with a gelatin solution diluted with $0.5 \%$ gadolinium contrast medium mixture. The stents were placed parallel to the $z$-axis of the MRI scanner; the scanning plane was basically axial.

\section{Magnetic susceptibility effect}

In the examination of magnetic susceptibility effect, measurements were performed with the stents aligned parallel to the $z$-axis and Band width (Bw) was changed from \pm 16.75 to $\pm 62.5 \mathrm{kHz} / \mathrm{FOV}$ to assess the influence of the $\mathrm{Bw}$ on stent-induced artifact. Also, TE was varied from 2.7 to $8.0 \mathrm{~ms}$ to assess the influence of the TE on stentinduced artifact. Carotid Wallstent measured the signal intensity (SI) at both the center and the end because both ends should have large artifacts. In-stent signal attenuation was assessed by calculating the relative in-stent signal (RIS) inside the stent. Artifact behavior of the stents was analyzed by measuring the SNR at the stent positions and compared with SNR measurements outside of the stents. Quantitative assessments were performed using approximately $78.5 \mathrm{~mm}^{2}$ (diameter: $10 \mathrm{~mm}$ ) ROI drawing in vertical to stents (Fig. 1).
The RIS $_{\text {stent }}$ indicated the severity of the artifact that decreased the SI inside a stent, respectively, and were calculated using Eq. (1)

$$
\mathrm{RIS}_{\text {stent }}=\left(\frac{\frac{\mathrm{SI}_{\text {in-stent }}}{\mathrm{SI}_{\mathrm{BG}}}}{\frac{\mathrm{SI}_{\text {out-stent }}}{\mathrm{SI}_{\mathrm{BG}}}}\right)
$$

where $\mathrm{SI}_{\text {in-stent }}, \mathrm{SI}_{\text {out-stent, }}$ and $\mathrm{SI}_{\mathrm{BG}}$ indicate the $\mathrm{SI}$ inside the stent, outside the stent, and that of the background area (consisting of air) on the 3D-TOF-MRA images. The SI was semi-automatically measured by means of the ImageJ.

RIS $_{\text {stent }}$ is defined such that 0 represents total signal loss and 1 represents no signal loss, where $\mathrm{SI}_{\text {out-stent }}$ is the signal in the area unaffected by the stents in the uniform phantom.

Fixed parameters in 3D-TOF-MRA were as follows: FOV: $120 \times 120 \mathrm{~mm}^{2}$, matrix: $256 \times 256$, TR: $25 \mathrm{~ms}$, flip angle (FA): $30^{\circ}$, slice thickness: $4.0 \mathrm{~mm}$, slice number: 16 , number of excitations (NEX): 1, acquisition time: $1 \mathrm{~min} 46 \mathrm{~s}$.

\section{RF-shielding effect}

Measurements were performed with the stents aligned parallel to the $z$-axis and only the FA was changed from 5 to $90^{\circ}$ with $5^{\circ}$ steps to assess the influence of the FA on stentinduced artifact. Quantitative assessments were performed using approximately $78.5 \mathrm{~mm}^{2}$ ROI drawing in each vertebra from all image sets. The change in Ernst angle was evaluated by measuring the $\mathrm{SI}_{\text {in-stent }}$ and $\mathrm{SI}_{\text {out-stent }}$ the stent for each FA.

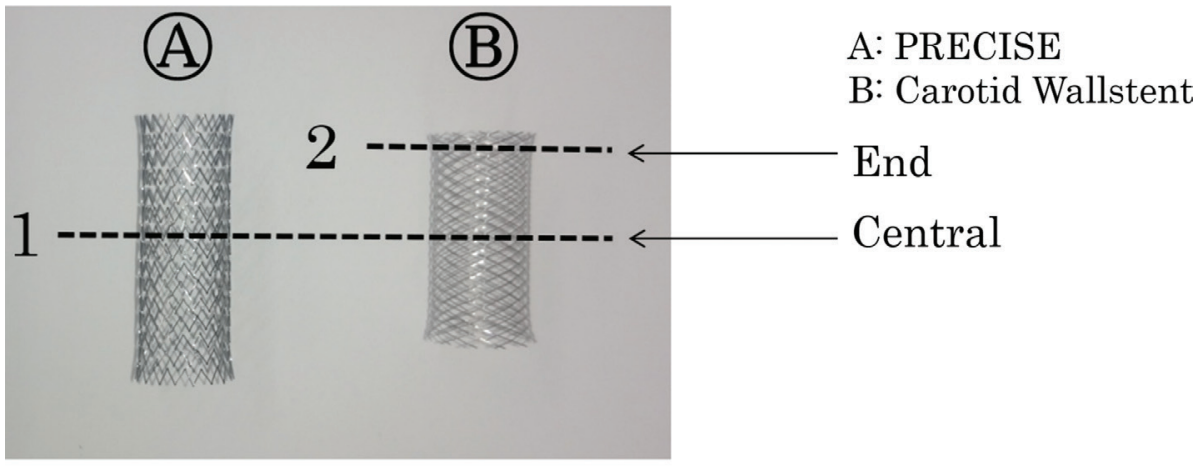

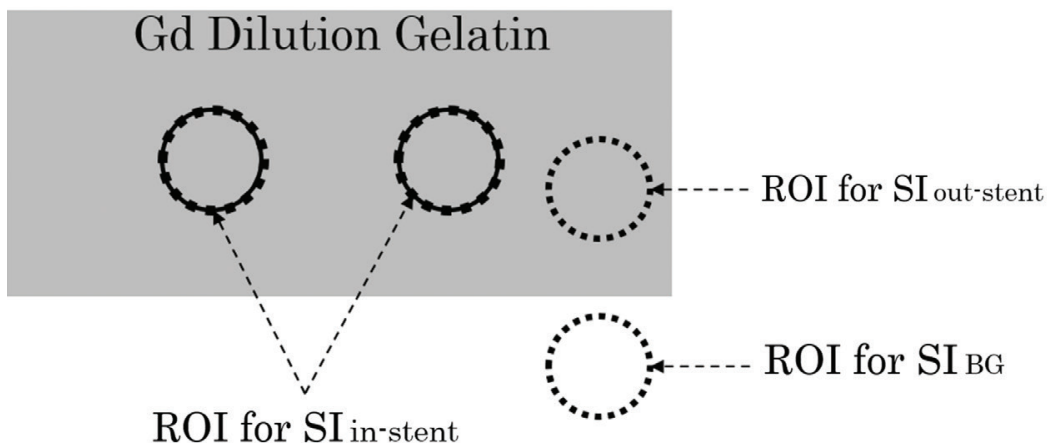

ROI size: $78.5 \mathrm{~mm}^{2}$
Fig. 1 Schematic of the phantom used in the measurements of magnetic susceptibility effect and RF-shielding effect. Locations of the ROls used for measurements of the luminal areas and the SI. SI, signal intensities. 
Fixed parameters in 3D-TOF-MRA were as follows: FOV: $120 \times 120 \mathrm{~mm}^{2}$, Matrix: $256 \times 256$, TR: $20 \mathrm{~ms}$, TE: $2.7 \mathrm{~ms}$, Bw: $\pm 62.5 \mathrm{kHz} / \mathrm{FOV}$, slice thickness: $4.0 \mathrm{~mm}$, slice number: 16, NEX: 1, acquisition time: $1 \min 26 \mathrm{~s}$.

\section{Clinical study}

\section{Patients}

The Clinical Ethics Committee of our hospital approved this study, and subjects were consented by participation. Subjects were consecutive patients (Carotid Wallstent: 4 men (4 regions), 2 women (2 regions); aged 61-82 years; mean age 76.4 years, PRECISE: 7 men (8 regions), 3 women (3 regions); aged 58-80 years; mean age 69.9 years) underwent MRI of CAS for cases with vulnerable plaque at our institution from January 2018 to April 2019. In this clinical study, the ability to visualize in-stent blood vessels was evaluated between images scanned under conventional imaging parameters and those scanned under optimal imaging parameters for each stent. Optimized imaging parameters were determined from the results of the basic experiment in PRECISE and Carotid Wallstent, respectively.

\section{Image assessment}

Using the most recent CTA image as the gold standard, one radiologist and three radiographers scored the image quality evaluation of the 3D-TOF-MRA of conventional and optimized imaging parameters, respectively, and calculated the average value of all evaluator data.

The following 3-point grading scales of visual evaluation were performed using CTA as a gold standard.

3-point: The inside of the stent is visualized and can be sufficiently diagnosed.

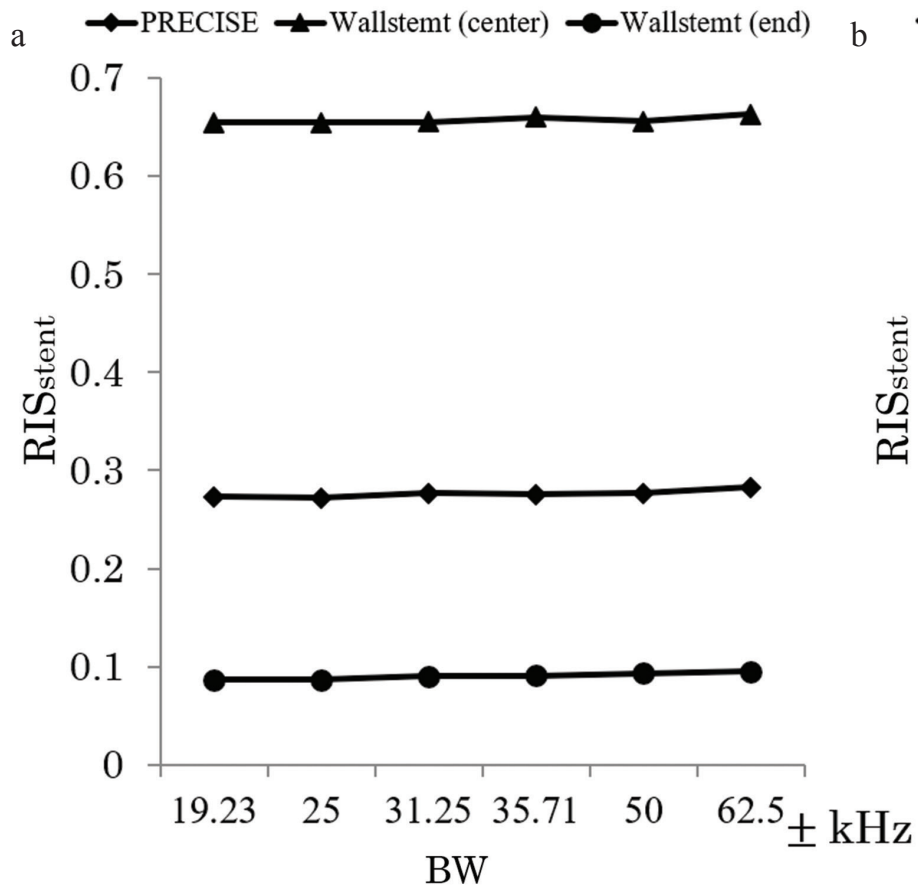

2-point: The inside of the stent is visible but not sufficient for diagnosis.

1-point: The inside of the stent is not visible due to artifacts.

The difference of mean score between conventional imaging parameters and optimized imaging parameters were assessed with Wilcoxon signed-rank test, where $P<0.01$ is considered statistically significant.

\section{Results}

\section{Phantom study}

Figure 2 shows the signal intensity exhibited by the gelatin solution diluted with $0.5 \%$ gadolinium contrast medium

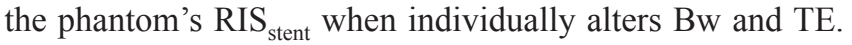
The experimental results of Bw show that the RIS stent $_{\text {at }}$ at the ends portion of the Carotid Wallstent was the lowest, followed by PRECISE, and the center of the Carotid Wallstent was the highest. Also, RIS stent $_{\text {between the obtained }}$ images was not affected by Bw. The experimental results

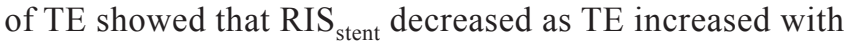
a particularly large reduction of about $57 \%$ at the ends portion of the Carotid Wallstent. In the next experiment, we used the same materials for the phantom as same as used in the magnetic susceptibility experiment. Figure 3 shows the result of RF-shielding effect. The Ernst angle of the Carotid Wallstent is $40^{\circ}$, the same as the outside of the stent, whereas $75^{\circ}$ in PRECISE. The magnetic susceptibility effect is small at the center of PRECISE and the Carotid Wallstent and greater at the end of the Carotid Wallstent. The RF-shielding effect was large at PRECISE

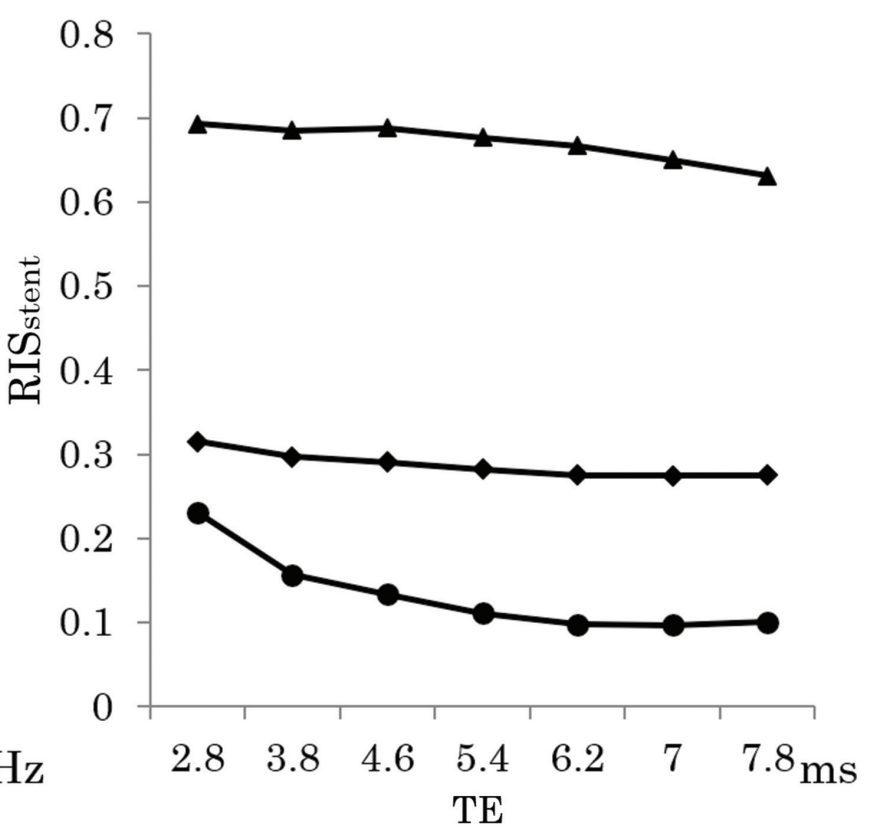

Fig. 2 The effects of the Bw and TE angle on the relative in-stent signal. (a) and (b) indicate the effects of Bw and TE. Bw, Band width. 


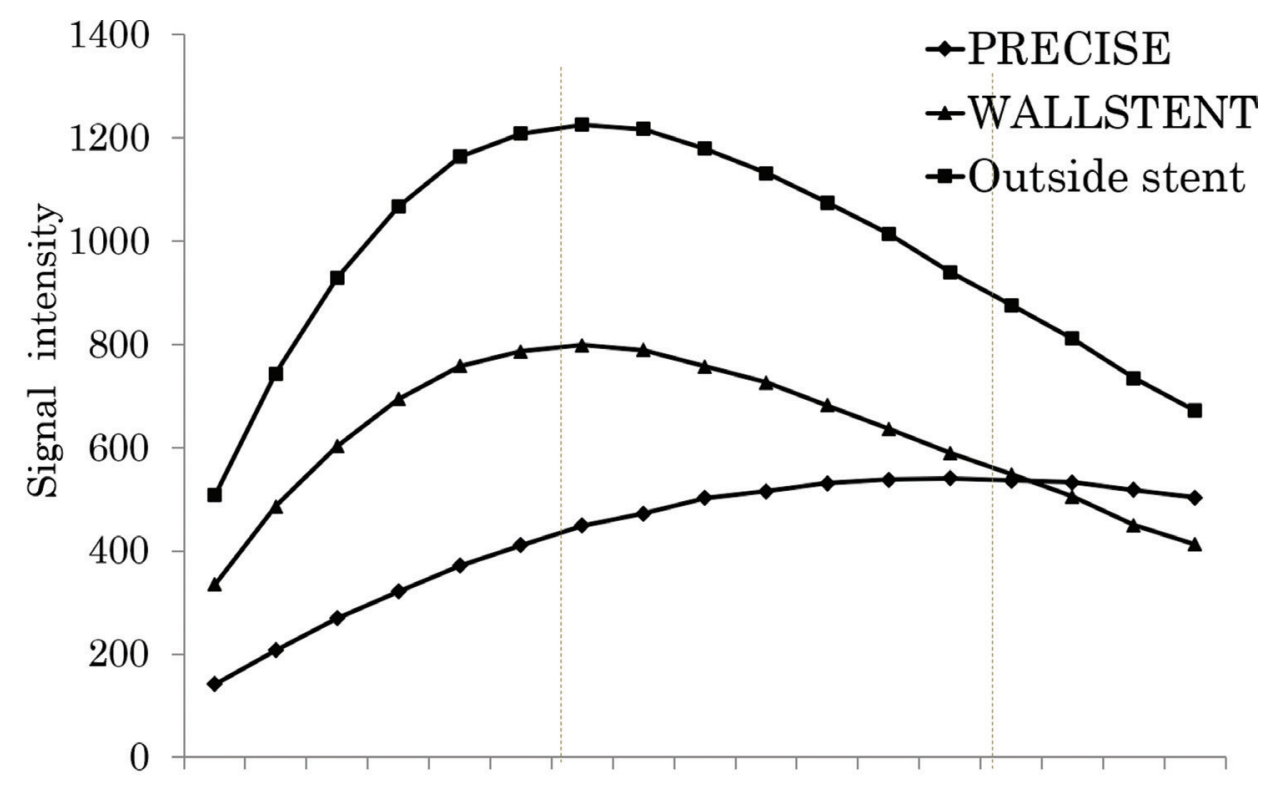

$\begin{array}{lllllllllllllllll}10 & 15 & 20 & 25 & 30 & 35 & 40 & 45 & 50 & 55 & 60 & 65 & 70 & 75 & 80 & 85 & 90\end{array}$ Flip angle
Fig. 3 The effects of the flip angle on in- and out-stent signal. Dashed lines indicate Ernst angle of each stent.

Table 1 Imaging parameters

\begin{tabular}{|c|c|c|c|}
\hline Sequence & $\begin{array}{c}\text { Conventional } \\
\text { parameters }\end{array}$ & $\begin{array}{c}\text { Optimized imaging } \\
\text { parameters of Wallstent }\end{array}$ & $\begin{array}{c}\text { Optimized imaging } \\
\text { parameters of PRECISE }\end{array}$ \\
\hline $\mathrm{TR}(\mathrm{ms})$ & 21 & 25 & 25 \\
\hline Flip angle $\left(^{\circ}\right)$ & 20 & 25 & 45 \\
\hline $\mathrm{TE}(\mathrm{ms})$ & 6.9 & 1.3 & 1.3 \\
\hline Matrix size & $256 \times 128$ & $224 \times 192$ & $224 \times 192$ \\
\hline $\mathrm{FOV}(\mathrm{mm})$ & 260 & 240 & 240 \\
\hline Slice thickness $(\mathrm{mm})$ & 2.0 & 1.6 & 1.6 \\
\hline Bandwidth of FOV $(\mathrm{kHz})$ & \pm 63 & \pm 63 & \pm 63 \\
\hline Flow compensation & On & Off & Off \\
\hline Number of excitations & 1 & 2 & 2 \\
\hline Number of slice & 118 & $24-36$ & $24-36$ \\
\hline Number of slub & 4 & 1 & 1 \\
\hline Acquisition time & $4 \min 48 s$ & $3 \min 25 \mathrm{~s}$ to $4 \mathrm{~min} 26 \mathrm{~s}$ & $3 \min 25 \mathrm{~s}$ to $4 \min 26 \mathrm{~s}$ \\
\hline Parallel factor & 2 & 1 & 1 \\
\hline
\end{tabular}

where the Ernst angle was greatly shifted and almost not at Carotid Wallstent.

\section{Optimization of imaging parameters setting of 3D-TOF-MRA}

Table 1 shows the conventional and the optimized imaging parameters. As a result of the phantom experiment, imaging parameters needed to be optimized for each type of stent. The imaging parameters for the Carotid Wallstent adopted a configurable minimum TE $1.3 \mathrm{~ms}$ because the magnetic susceptibility effect was so large that the TE needed to be shortened. In addition, it is necessary to use large $\mathrm{Bw}$ to reduce TE, which results in deteriorating SNR. Therefore, we extended TR and used FA of $25^{\circ}$ to compensate SNR. The imaging parameters for the PRECISE adopted a maximum of $45^{\circ}$ that can be set when using ramped RF-pulses, because the RF-shielding effect is very large and the FA needs to be deepened. 


\section{Clinical study}

In the results of Carotid Wallstent, the score of conventional imaging parameters was $1.96 \pm 0.04$ and the score of optimized imaging parameters was $2.63 \pm 0.24$. The score of optimized imaging parameters was about $34 \%$ higher than conventional imaging parameters (Fig. 4a); in addition, the difference of mean score was statistically significant using Wilcoxon signed-rank test $(P<0.01)$. Optimized imaging parameters could significantly reduce the magnetic susceptibility artifacts in the proximal part of Carotid Wallstent. However, the distal artifact did not completely improve in all cases of the Carotid Wallstent (Fig. 5).

In the results of PRECISE, the score of conventional parameters was $1.00 \pm 0$ and the score of optimized imaging parameters was $2.72 \pm 0.20$. The score of optimized imaging parameters was expressly higher than conventional imaging parameter (Fig. 4b); in addition, the difference of mean score was statistically significant using Wilcoxon signed-rank test $(P<0.01)$. Optimized imaging parameters could significantly reduce the RF-shielding artifact of PRECISE (Fig. 6) improved in all cases of PRECISE, but not enough in some cases. In the case where multiple stents were used, the signal decreased due to the increased shielding effect in the overlapping part, and the signal decreased in the distal part because in-flow effect could not be secured (Fig. 7a).

Also, in lesions with high residual stenosis due to severe calcification, turbulence caused pseudo-stenosis distal to the stenosis (Fig. 7b).

$\mathrm{a}$

Carotid Wallstent

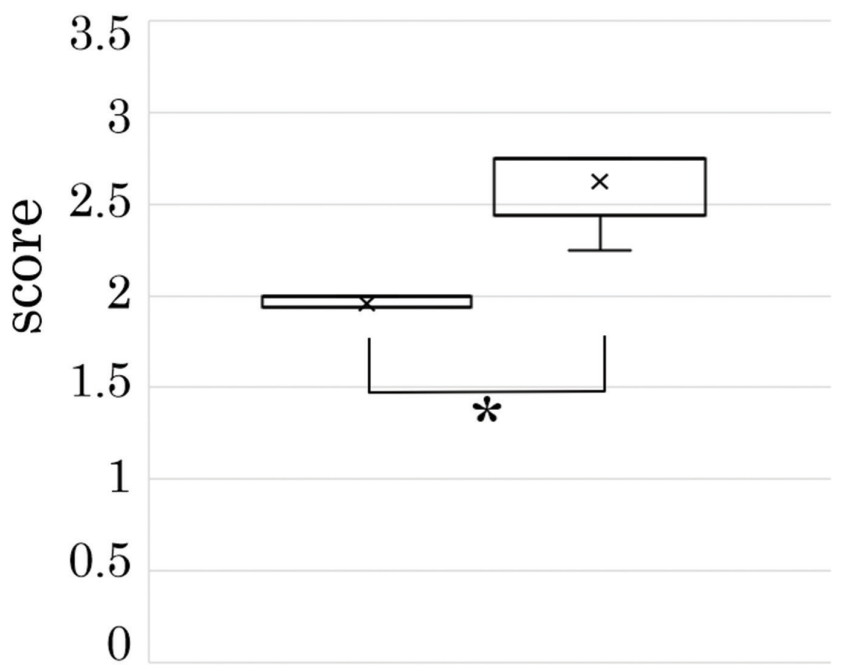

\section{Discussion}

In the present study, we investigated the causes of artifacts and their countermeasures on 3D TOF-MRA images of posterior carotid stenting. Consequently, it was revealed that the influence of the magnetic susceptibility effect, the RFshielding effect and the setting of the optimal imaging parameters varied depending on the type of carotid stent. Several studies have investigated the relationship between imaging parameters and stent-induced artifacts and the relationship has been reported to be due to material and stent design..$^{7-9}$ However, their imaging parameters were not deeply considered. Therefore, we believe that our results could help for optimizing imaging parameters in clinical practice. As mentioned in the introduction, there are two kinds of artifacts induced by a stent, one is the magnetic susceptibility artifact and the other is the RF-shielding artifact. It is also reported that wide $\mathrm{Bw}$ and short $\mathrm{TE}$ are effective in suppressing magnetic susceptibility artifacts, ${ }^{10}$ but this is the case of setting to automatically minimize TE. In our study, according to a phantom experiment in which $\mathrm{Bw}$ and TE were independently changed, RIS stent $_{\text {was reduced by shortening TE, but }}$ there was no correlation with $\mathrm{Bw}$. The magnetic susceptibility effect has distortion and signal loss, and we consider

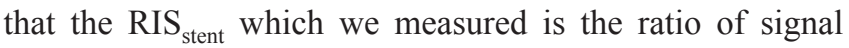
intensity, so the distortion due to Bw change did not affect it. In the clinical studies of Carotid Wallstent, signal loss was caused by strong magnetic susceptibility artifacts at both ends under conventional imaging parameters, but improved

$\mathrm{b}$

PRECISE

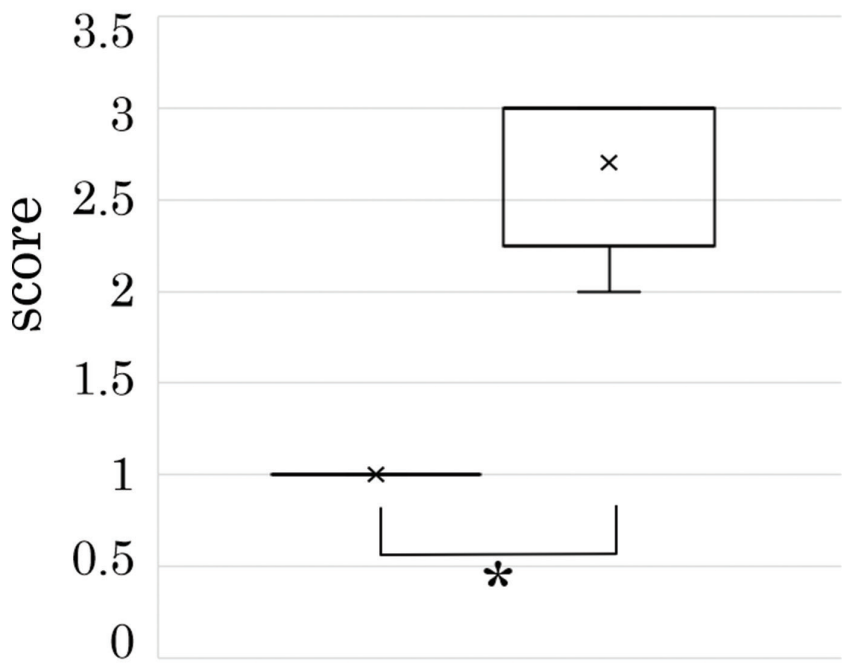

\section{conventional optimize}

\section{conventional optimize}

Fig. 4 Subjective analysis of visibility of 3D-TOF-MRA of post-carotid artery stenting. (a) Carotid Wallstent, (b) PRECISE. In (a) and (b), the asterisks $\left(^{*}\right)$ mean "significant differences" using Wilcoxon signed-rank test. 3D-TOF-MRA, 3D time-of-flight MR angiography. 

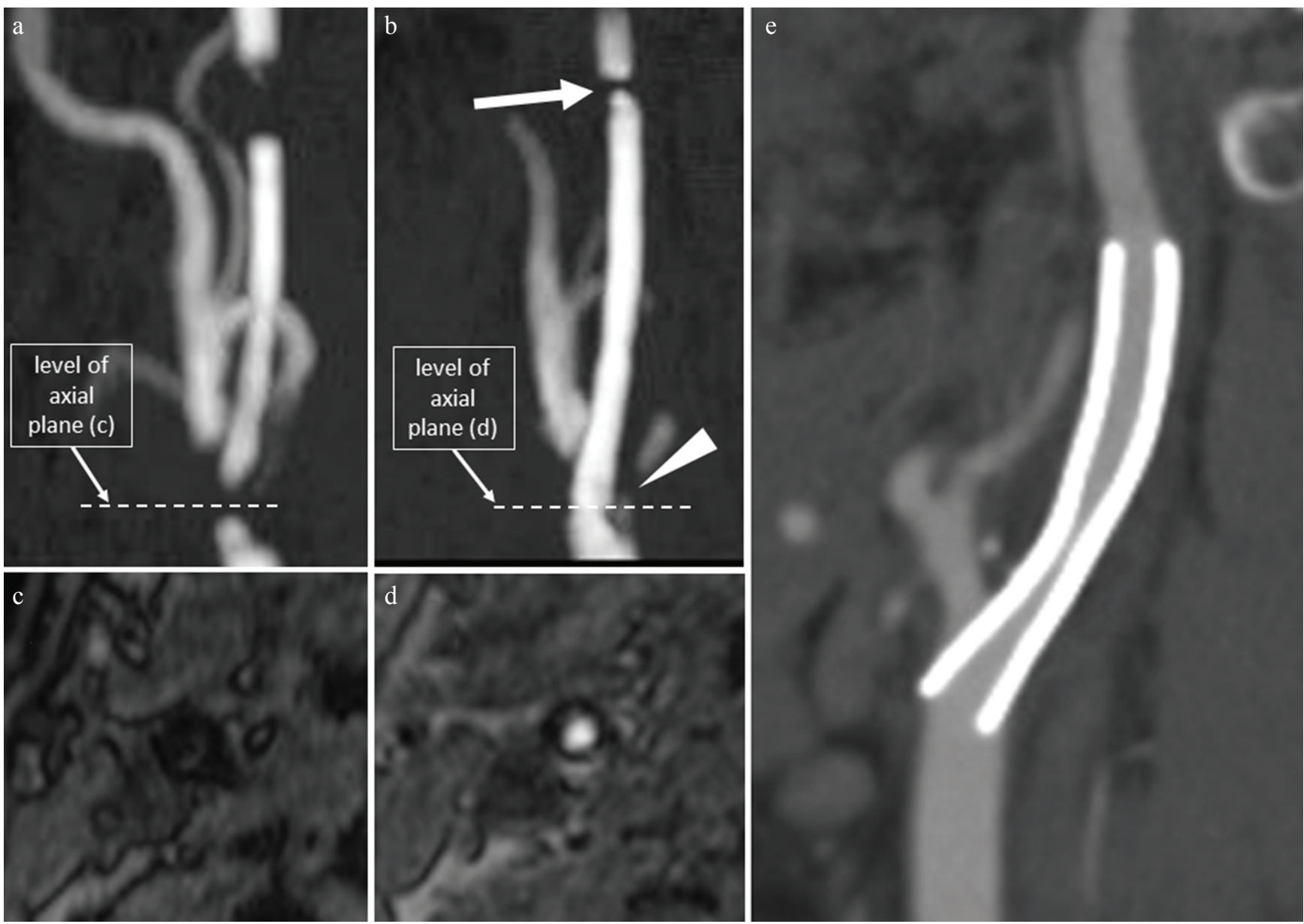

Fig. 5 Post-carotid artery stenting (Wallstent $8 \mathrm{~mm} / 21 \mathrm{~mm}$ ) in an 80-years-old woman. (a) MIP of conventional imaging parameters. (b) MIP of optimized imaging parameters. (c) Axial of conventional imaging parameters. (d) Axial of optimized imaging parameters. (e) CPR of CTA. Poor improvement in distal artifact (b, arrow) compared with artifact (b, arrow head) in the proximal portion of the Wallstent. CPR, curved planer reconstruction; CTA, computed tomography angiography; MIP, maximum intensity projection.

under optimized imaging parameters. However, the distal portion remained partially lost. Although the magnetic susceptibility artifacts of cobalt alloy stents were reported to be lower than that of most nitinol stents, they had a strong signal loss in the both ends of Carotid Wallstent. ${ }^{11}$ We consider that the signal loss at both ends of Carotid Wallstent is not due to material. Because RIS in PRECISE (nitinol) was lower than that in the Carotid Wallstent (cobalt-chromium alloy, tantalum) on the center of stents. For this reason, we paid attention to the design of both ends of the Carotid Wallstent. We consider that the wires at both ends of the stent other than the Carotid Wallstent are closed loop, and that eddy currents may be generated because only the Carotid Wallstent is open loop (Fig. 8). Also, the artifacts differ between the proximal and distal parts. It is reported that the magnetic susceptibility artifacts depend on the magnetic susceptibility of the stent relative to the surrounding tissue, on the orientation of the stent relative to the static magnetic field. ${ }^{12}$ It is speculated that this light may be due to the direction of the static magnetic field and the direction of application of the gradient magnetic field. However, with CTA in Carotid Wallstent artifacts such as exaggerated thickening or blooming of the stent struts, are well known resulting narrowing of the stents. Therefore, MRA is not perfect but is useful for follow-up of Carotid Wallstent. In the RF-shielding effect phantom experiment, the RF-shielding effect was large in PRECISE, and the Ernst angle differed $35^{\circ}$ between the inside and outside of the stent. Therefore, $55^{\circ}$ which is actually $35^{\circ}$ higher than the normal state, is the optimal FA. However, we adopted FA $45^{\circ}$ which is $25^{\circ}$ larger than the conventional imaging parameters. This is because the limit of $\mathrm{FA}$ on this equipment is $45^{\circ}$ when using ramped RF-pulses to equalize proximal and distal vascular signals. In this study, we revealed that the effect of the magnetic susceptibility effect and the RF-shielding effect and the setting of the optimal imaging parameters were different depending on the type of carotid stent. Earlier reports show that the number of wires in braided stents and stent design can affect the shielding effectiveness. ${ }^{13}$ Other reports show that the shielding effects varied with stent materials, geometry, and orientation. ${ }^{15}$ Experiments were performed by using intracranial stents (Enterprise [Cordis, Miami, FL, USA], LVIS Jr [Terumo, Tokyo, Japan], ATLAS [Stryker, 

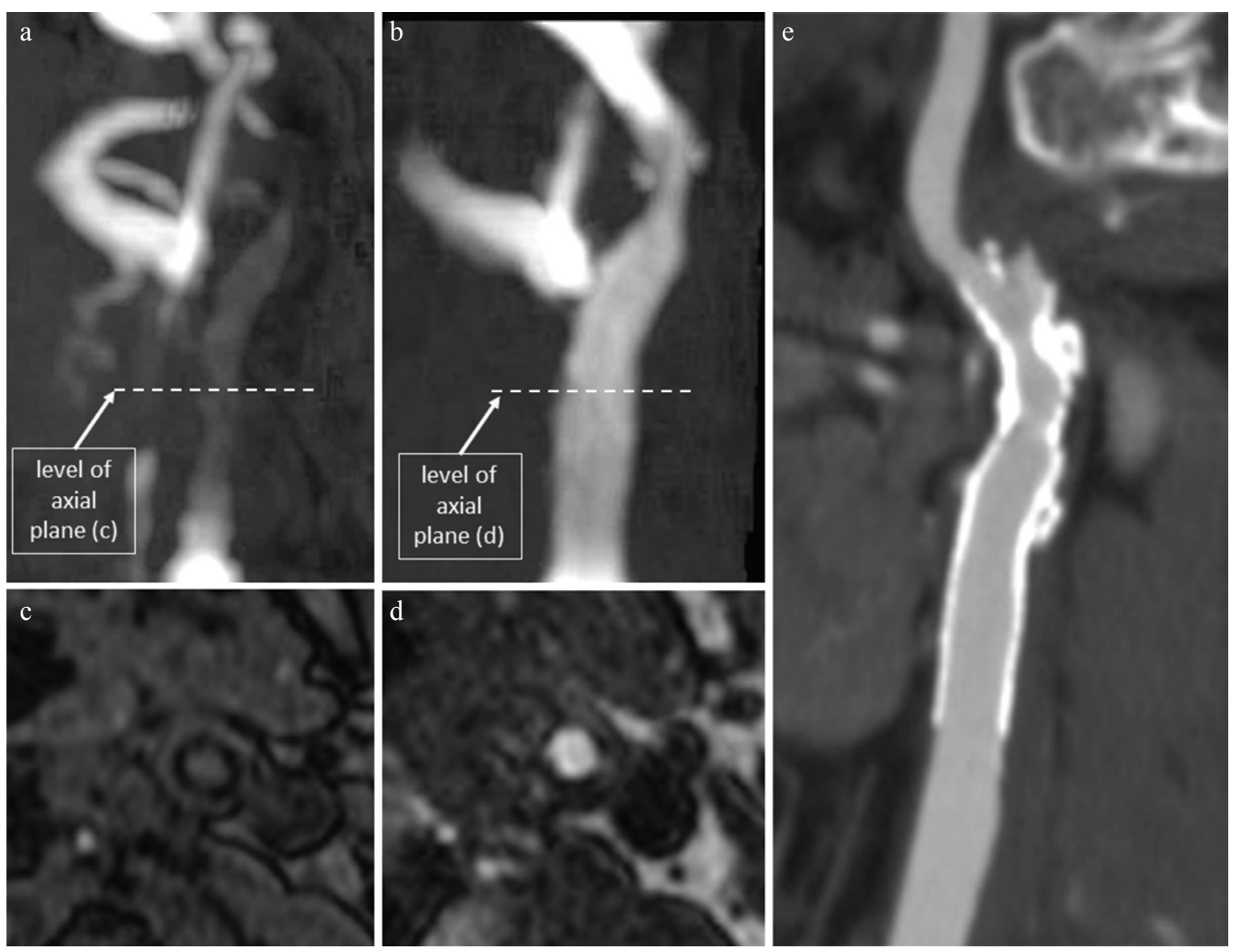

Fig. 6 Post-carotid artery stenting (PRECISE $7 \mathrm{~mm} / 40 \mathrm{~mm}$ ) in a 70-years-old woman. (a) MIP of conventional imaging parameters. (b) MIP of optimized imaging parameters. (c) Axial of conventional imaging parameters. (d) Axial of optimized imaging parameters. (e) CPR of CTA. CPR, curved planer reconstruction; CTA, computed tomography angiography; MIP, maximum intensity projection.
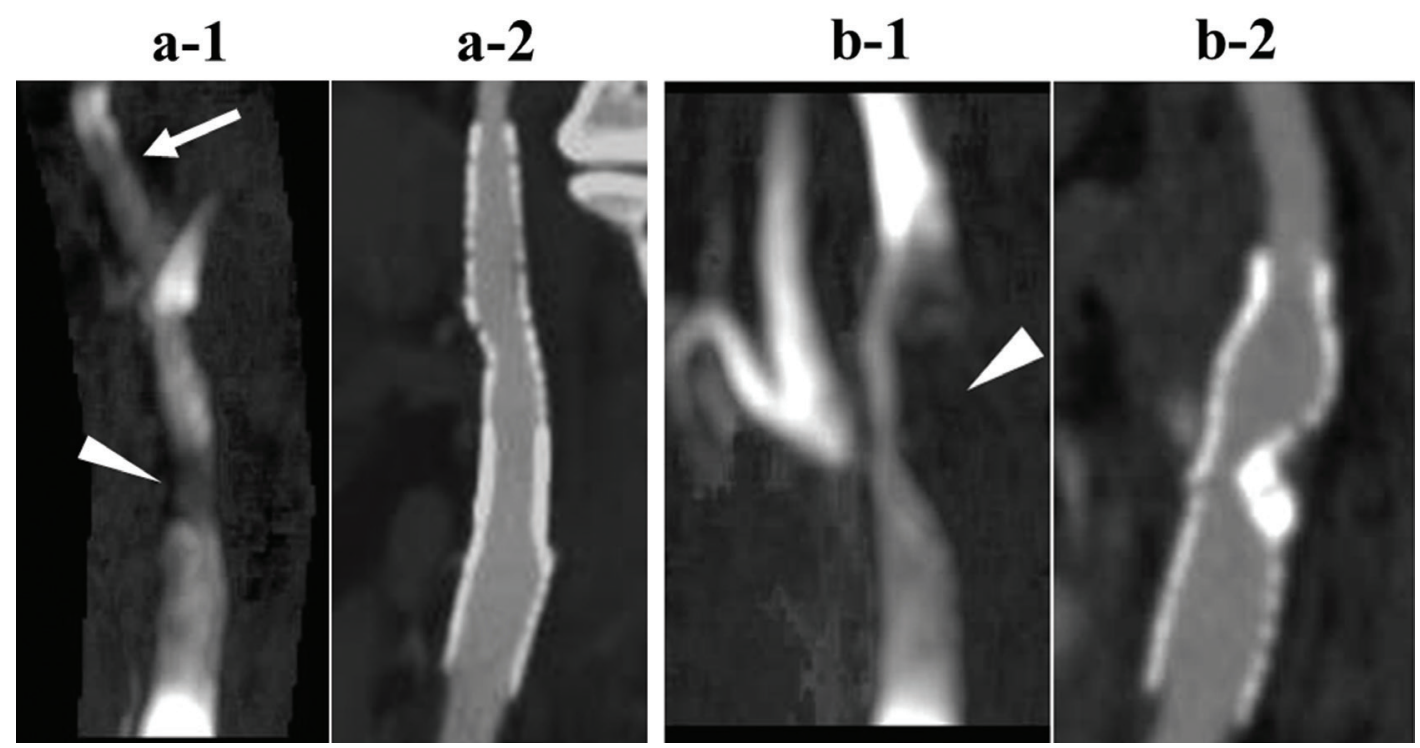

Fig. 7 (a) Post-CAS (PRECISE $8 \mathrm{~mm} / 40 \mathrm{~mm}$, PRECISE $10 \mathrm{~mm} / 30 \mathrm{~mm}$ ) in a 59-years-old man. (a-1) MIP of optimized imaging parameters. (a-2) CPR of CTA. (b) Post-CAS (PRECISE) in a 63-years-old man. (b-1) MIP of optimized imaging parameters. (b-2) CPR of CTA. In lesions with high residual stenosis, turbulence caused pseudo-stenosis distal to the stenosis (b-1, arrow head). The signal was reduced with an increased shielding effect in the overlapping part (a-1, arrow head). The signal was reduced with a decrease in-flow effect of the distal part (a-1, arrow). CAS, carotid artery stenting; CPR, curved planer reconstruction; CTA, computed tomography angiography; MIP, maximum intensity projection. 

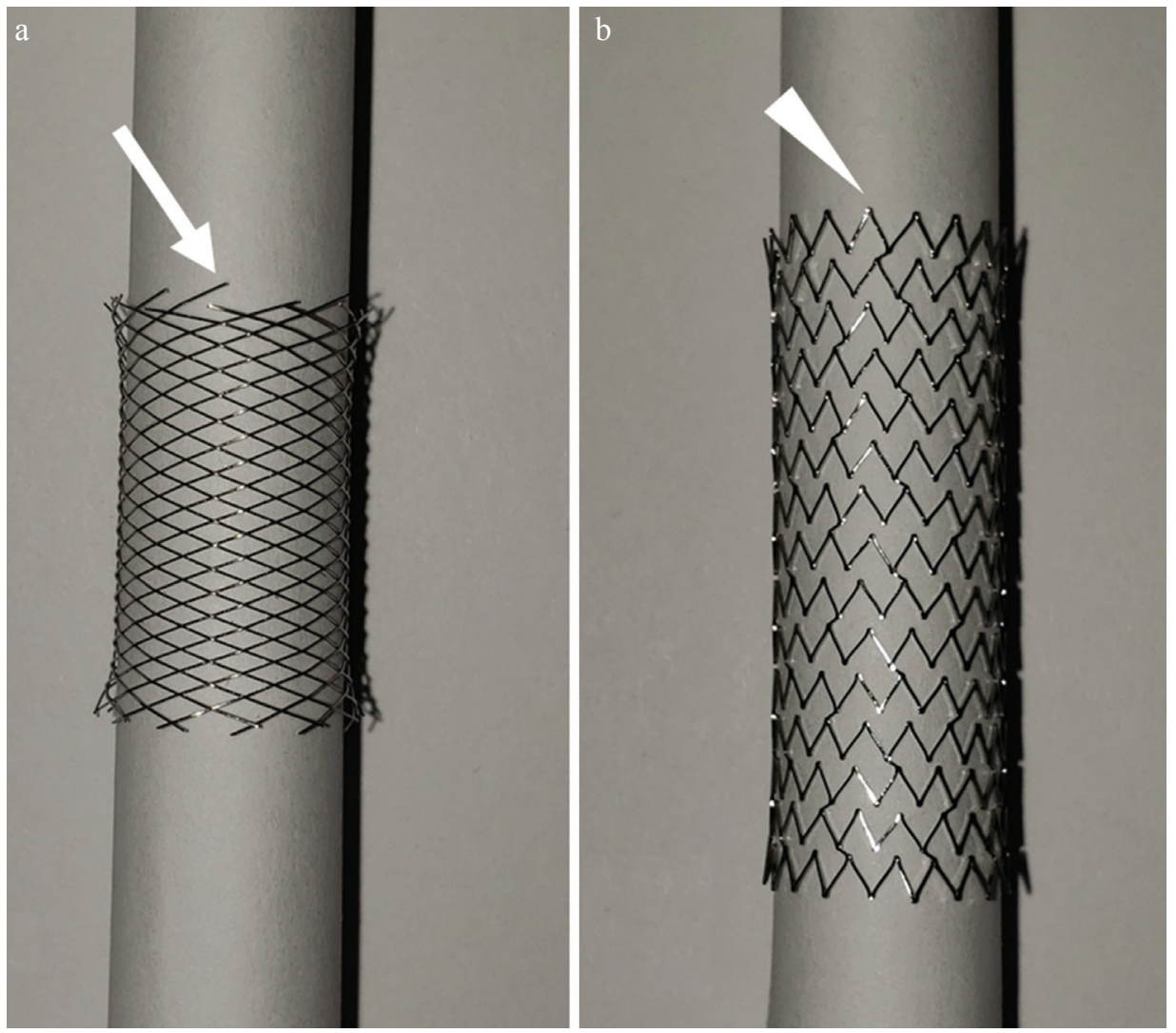

Fig. 8 Photographs of stents. (a) Carotid Wallstent. (b) PRECISE. The wires at both ends of the Carotid Wallstent is open loop (arrow) and the PRECISE is closed loop (arrow head).

Kalamazoo, MI, USA]) for the purpose of investigation of the relationship between RF-shielding effect and material and design. An experiment of RF-shielding effect using intracranial stents showed that the RF-shielding effect of Enterprise was the largest, ATLAS was moderate, and LVIS $\mathrm{Jr}$ was very small. According to the results of the material, cell type, and RF-shielding effect of the two types of carotid stents and three types of intracranial stents, there was no correlation between the RF-shielding effect and material, and cell type. We consider that the factor may be due to the quantity of metallic material per unit area and stent design, but we have not reached a conclusion. In the past, the evaluation of the stent site post-CAS has mainly been reported using contrast agents such as CTA. However, by optimizing imaging parameters of 3D-TOF-MRA this time, imaging became possible without using a contrast agent. ${ }^{15,16}$ Our study was performed in vitro with a static phantom design and in vivo with a clinical case. As mentioned in the introduction, there are two kinds of artifacts induced by a stent, one is the magnetic susceptibility artifact and the other is the RF-shielding artifact. The former can be improved by using a short TE, and the latter can be decreased by using high FA. ${ }^{17-21}$ Our results will be helpful in determining the parameters for 3D-TOFMRA because our study clarifies the effect of the devices on image quality. Although we have investigated only two kinds of stents in this work, we expect that true cause of signal drop would be revealed through investigation of many kinds of stents in the future.

\section{Conclusion}

By analyzing the 3D-TOF-MRA artifacts caused by a representative two types of stents, we could identify the major role of the magnetic susceptibility effect and RF-shielding effect. Furthermore, the minimizing the TE of 3D TOF-MRA was reduced magnetic susceptibility artifacts are reduced in Carotid Wallstent, and the optimization of the FA may reduce RF-shielding artifacts in PRECISE, rendering feasible the 3D-TOF-MRA. Consequently, visibility of the stent lumen was improved and diagnostic reliability of 3D-TOF-MRA was markedly increased. Hence, we demonstrated that our optimized imaging parameters markedly improved the ability to visualize such lesions.

\section{Conflicts of Interest}

The authors declare that they have no conflicts of interest

\section{References}

1. Kono K, Shintani A, Terada T. Non-enhanced magnetic resonance angiography can evaluate restenosis after carotid artery stenting with the Carotid Wallstent. Acta Neurochir (Wein) 2014; 156:1713-1719.

2. Debrey SM, Yu H, Lynch JK, et al. Diagnostic accuracy of magnetic resonance angiography for internal carotid artery disease: a systematic review and meta-analysis. Stroke 2008; 39:2237-2248. 
3. Lövblad KO, Yilmaz $\mathrm{H}$, Chouiter $\mathrm{A}$, et al. Intracranial aneurysm stenting: follow-up with MR angiography. J Magn Reson Imaging 2006; 24:418-422.

4. Choi JW, Roh HG, Moon WJ, et al. Time-resolved 3D contrast-enhanced MRA on 3.0T: a non-invasive followup technique after stent-assisted coil embolization of the intracranial aneurysm. Korean J Radiol 2011; 12:662-670.

5. Duerig TW, Tolomeo DE. An Overview of Superelastic Stent Design. In: Russell SM, Pelton AR, eds. Proceedings of the International Conference on Shape Memory and Superelastic Technologies (SMST), TIPS Technical Publishing Inc., California: 2001; 585-604.

6. Teitelbaum GP, Bradley WG, Klein BD. MR imaging artifacts, ferromagnetism, and magnetic torque of intravascular filters, stents and coils. Radiology 1988; 166:657-664.

7. Wang Y, Truong TN, Yen C, et al. Quantitative evaluation of susceptibility and shielding effects of nitinol, platinum, cobalt-alloy, and stainless steel stents. Magn Reson Med 2003; 49:972-976.

8. van Holten J, Wielopolski P, Brück E, Pattynama PM, van Dijk LC. High flip angle imaging of metallic stents: implications for MR angiography and intraluminal signal interpretation. Magn Reson Med 2003; 50:879-883.

9. Graf H, Klemm T, Lauer UA, Duda S, Claussen CD, Schick F. [Systematics of imaging artifacts in MRT caused by metallic vascular implants (stents)]. Rofo 2003; 175: 1711-1719 (in German).

10. Ikushima $Y$, Hashido $T$, Watanabe $Y$, Doi T. Effects of imaging parameters on the quality of contrast-enhanced MR angiography of cerebral aneurysms treated using stentassisted coiling: a phantom study. Magn Reson Med Sci 2017; 16:146-151.

11. Lettau M, Sauer A, Heiland S, Rohde S, Bendszus M, Hähnel S. Carotid artery stents: in vitro comparison of different stent designs and sizes using CT angiography and contrast-enhanced MR angiography at 1.5T and 3T. AJNR Am J Neuroradiol 2009; 30:1993-1997.

12. Bakker CJ, Bhagwandien R, Moerland MA, Ramos LM. Simulation of susceptibility artifacts in 2D and 3D Fourier transform spin-echo and gradient-echo magnetic resonance imaging. Magn Reson Imaging 1994; 12:767-774.

13. Bouillot $P$, Brina $O$, Delattre BMA, et al. Neurovascular stent artifacts in 3D-TOF and 3D-PCMRI: influence of stent design on flow measurement. Magn Reson Med 2019; 81:560-572.

14. Schenck JF. The role of magnetic susceptibility in magnetic resonance imaging: MRI magnetic compatibility of the first and second kinds. Med Phys 1996; 23:815-850.

15. Frölich AM, Pilgram-Pastor SM, Psychoqios MN, Mohr A, Knauth M. Comparing different MR angiography strategies of carotid stents in a vascular flow model: toward stent-specific recommendations in MR follow-up. Neuroradiology 2011; 53:359-365.

16. Borisch I, Hamer OW, Zorger N, Feuerbach S, Link J. In vivo evaluation of the carotid wallstent on threedimensional contrast material-enhanced MR angiography: influence of artifacts on the visibility of stent lumina. J Vasc Interv Radiol 2005; 16:669-677.

17. Bartels LW, Smits HF, Bakker CJ, Viergever MA. MR imaging of vascular stents: effects of susceptibility, flow, and radiofrequency eddy currents. J Vasc Interv Radiol $2001 ; 12: 365-371$.

18. Holton A, Walsh E, Anayiotos A, Pohost G, Venugopalan R. Comparative MRI compatibility of 316 L stainless steel alloy and nickel-titanium alloy stents. J Cardiovasc Magn Reson 2002; 4:423-430.

19. Meyer JM, Buecker A, Spuentrup E, et al. Improved instent magnetic resonance angiography with high flip angle excitation. Invest Radiol 2001; 36:677-681.

20. Straube T, Wolf S, Flesser A, et al. [MRI of carotid stents: influence of stent properties and sequence parameters on visualization of the carotid artery lumen]. Rofo 2005; 177:375-380 (in German).

21. Klemm T, Duda S, Machann J, et al. MR imaging in the presence of vascular stents: a systematic assessment of artifacts for various stent orientations, sequence types, and field strengths. J Magn Reson Imaging 2000; 12:606-615. 